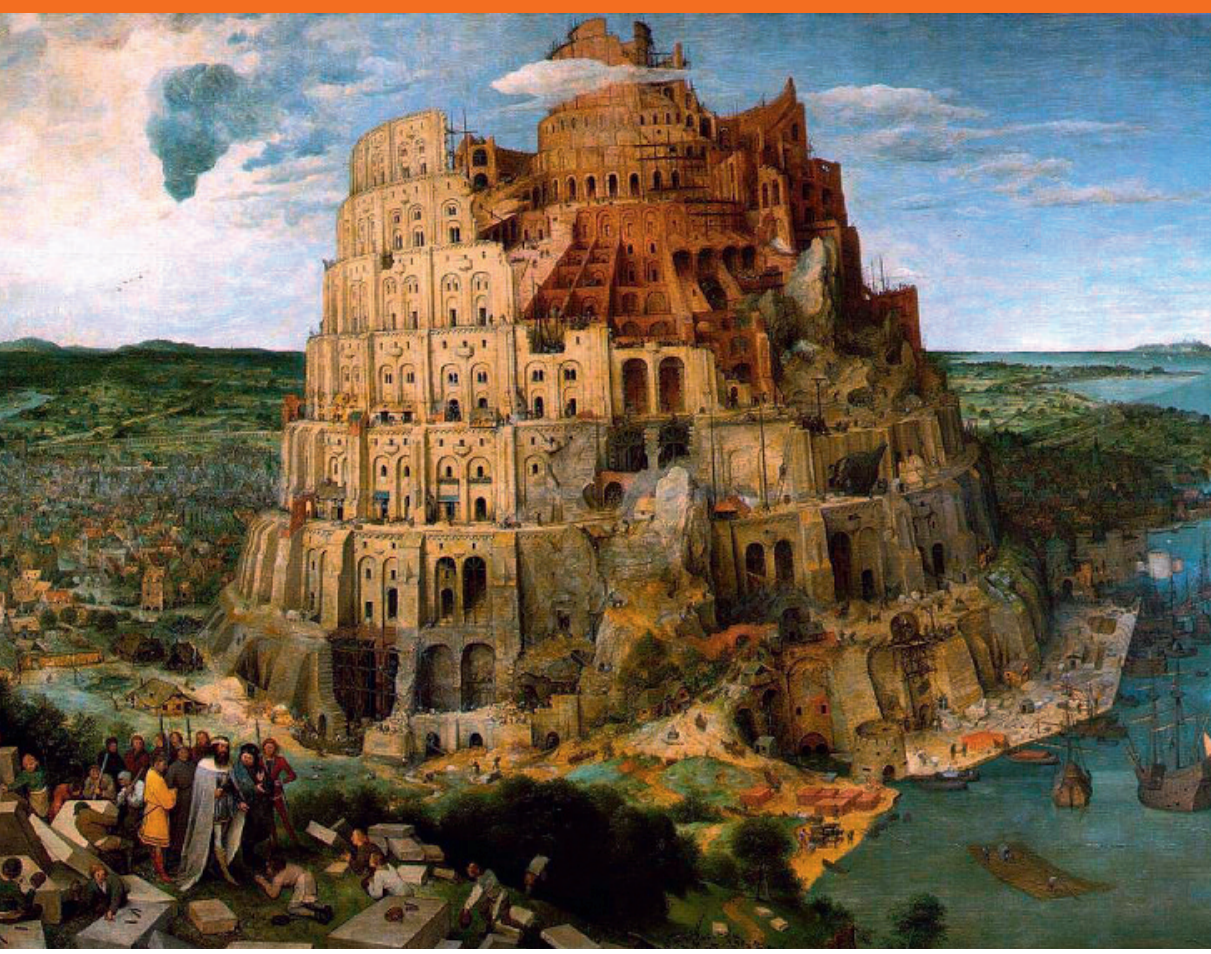

Werner Graf

Korrespondenz:

Dr. med. Werner Graf Holenackerstrasse 85 CH-3027 Bern

we.graf@bluewin.ch
Kennen Sie das berühmte Bild vom Turmbau zu Babel? Nein? Das Bild, das der grosse Brueghel, Pieter Brueghel de Oude, 1563 als Allegorie auf das menschliche Leben gemalt hat: Spitzenleistung oder Gotteslästerung oder einfach das Scheitern des Menschen?

Eine riesige Baustelle erhebt sich in den Himmel, ein irrwitziger Turm, eine unvollendete Pyramide, der oberste Teil noch im Bau, durchbricht die Wolkendecke, so dass jene, die oben am Bauen sind, die Erde nicht mehr sehen können, und jene, die weiter unten bauen, nicht mehr wissen, was oben geschieht.

Wer sind diese Leute? Es sind Handwerker, Steinhauer, Steinmetze, ein Heer von Lastenträgern und Gehilfen, Maurer, Schlosser und Schreiner, Spezialisten für Statik und kühne Konstruktionen, aber auch Künstler, manche von ihnen mit zwei goldenen Händen und einem feinen Gespür für Form und Stein. Daneben gibt es aber auch Planer und Organisatoren, Aufseher und Oberaufseher, Wesire, Muftis und Mogule. Wo aber gebaut wird, fliesst Geld. Also gibt es auch Schatzmeister, Oberschatzmeister, Säckelmeister und Obersäckelmeister, Verwalter der Kassen und Tresore, Schatzkämmerer, Berater, Buchhalter und wieder Berater sowie ein Heer von Zulieferern aller Art. Oberster Aufseher aber war der Grossmogul, der nicht nur durch seinen angeblich planerischen Weitblick und enormen Ideenreichtum, sondern auch durch seine grosse Nase auffiel, so dass später, als der Turmbau ins Stocken geriet, beim Sprichwort «an der Nase herumgeführt werden» jeder sofort an den Grossmogul dachte.

Indes, alle verdienten ihr Brot am Turmbau zu Babel und waren glücklich und zufrieden, wenn weitergebaut wurde, höher und höher. Je höher aber der Turm in den Himmel wuchs, desto mehr wuchs auch die Zahl der Steinmetze und Bauspezialisten. Die Zahl der Berater und Zulieferer schwoll aber noch mehr an, überproportional schwoll sie an, so dass sie zunächst

\section{Vom Turmbau zu Babel}

Parallelen zum Gesundheitswesen von heute? Pieter Brueghels «Turmbau zu Babel» aus dem Jahr 1563.

nur hinter vorgehaltener Hand, dann immer unverhohlener spöttisch als Trittbrettfahrer bezeichnet wurden. Wer wollte sich schon die Hände schmutzig machen? Wenn einem Steinmetz ein Fehler passierte, hiess es sogleich: Seht her, sie machen nichts als Fehler, man sollte ihnen besser auf die Finger schauen oder den Lohn kürzen. So wurde es immer schwieriger, junge Steinmetze zu finden, die noch bereit waren, den Hammer in die Hand zu nehmen. Ihr Beruf galt als unattraktiv. Planungsfehler dagegen konnten immer auf die nächsthöhere Instanz abgeschoben werden, bis zuletzt keiner mehr verantwortlich war. Der Grossmogul hat es so angeordnet, hiess es dann, und der schwebte über den Wolken, ihm konnte niemand etwas anhaben. L'état c'est moi.

Aber auch unter den Planern und Architekten gab es Zwietracht. Sagte der eine Hü, so sagte der andere Hott. Wollte ein Säckelmeister hier mehr investieren, wollte der andere lieber dort. Jeder hatte seine eigenen Vorstellungen, wie weitergebaut werden sollte, wo wie gespart werden und wo mehr investiert werden sollte. Jeder wusste es besser und dachte dabei nur an sich. Auf die Idee, man könnte vielleicht die Steinmetze und Künstler mit in die Planung einbeziehen, kam niemand. Der Bau geriet ins Stocken.

Wieso erzähle ich diese Geschichte? Ich erzähle sie, weil sie mich an die heutige Medizin erinnert: immer höher und besser, immer gesünder und immer schneller gesünder, immer mehr und immer noch mehr. Jeder bereichert sich auf seine Weise, profitiert, wo er kann, und fordert gleichzeitig, dass zwar die Menge, nicht aber deren Kosten ausgeweitet werden. Dazu kommt, wie beim Turmbau zu Babel, eine weitere Verwirrung: die Frage nach Sinn und Unsinn des «Immer mehr». Wer bestimmt denn eigentlich, dass immer höher gebaut werden muss? Sind es wirklich die Steinmetze, die mit dem Hammer in der Hand, oder sind 
es am Ende die Trittbrettfahrer, die sekundären Nutzniesser?

Ich denke, dass es höchste Zeit ist, über eine zentrale Figur nachzudenken, die befähigt ist, einen vernünftigen Weg aus dem ins Stocken geratenen Turmbau zu Babel zu finden. Diese Person muss ein gewichtiges politisches Mitspracherecht haben. Der Grossmogul soll diese nicht nur anhören, sondern er soll auch zur Kenntnis nehmen, was sie ihm sagt, sie muss eine Ärztin oder ein Arzt sein (und nicht ein Jurist, wie im BAG!), die neben Fachkenntnissen auch über den sogenannten gesunden Menschenverstand verfügt, eine Eigenschaft, die nicht hoch genug eingeschätzt werden kann. Wer kommt in Frage? Ich denke, am ehesten ein Arzt oder eine Ärztin mit einer grossen praktischen Erfahrung.

\section{Buchbesprechung}

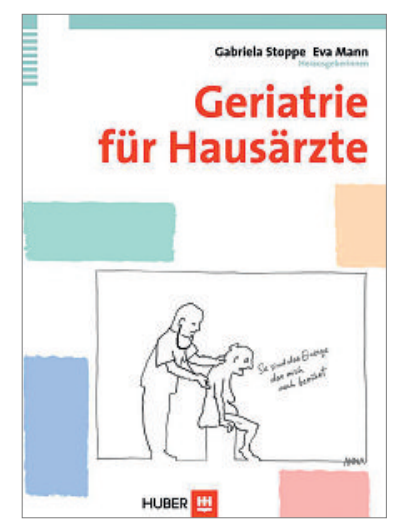

\section{Geriatrie}

Gabriela Stoppe, Eva Mann (Hrsg.)

\section{Geriatrie für Hausärzte}

Bern: Hans Huber; 2009

456 Seiten. Fr. 99 .-

ISBN 978-3-456-84705-4

Geriatrie und Gerontopsychiatrie sind ein weites Feld, das nicht nur den Spezialisten für Geriatrie überlassen bleibt, sondern alle Fachärzte und insbesondere Hausärzte betrifft. Nicht nur, dass es immer mehr alte Menschen gibt, sie werden auch immer älter und multimorbider. Das Buch von Gabriela Stoppe und Eva Mann stellt ein Praxishilfsmittel für Hausärzte dar, die sich der Herausforderung der Betreuung älterer Menschen annehmen. Es ist der Abklärung und Behandlung geriatrischer Patienten gewidmet.

Das grosse Autorenteam setzt sich aus Forschern und Ärzten verschiedener Fachrichtungen zusammen, die im gesamten deutschsprachigen Raum tätig sind. In dem Buch werden konkrete Empfehlungen und Richtlinien zum Vorgehen bei der Versorgung geriatrischer Patienten gegeben. Es gliedert sich in vier grosse The-
PS: Als aber dem Grossmogul dämmerte, dass das Klima kälter und sein Überblick beim Turmbau zu Babel lückenhaft zu werden begann, er einfache $\mathrm{Zu}$ sammenhänge nicht mehr zu erkennen vermochte und, wie böse Zungen behaupteten, schwadronierend über sie hinwegging, dachte er an seinen Rücktritt. Er erinnerte sich an die hochgelobte Formel, die andere Grossnasen vor ihm, in ähnlicher Zwickmühle steckend, auch schon zu Hilfe gerufen hatten, um sich elegant und für das gemeine Volk verdaubar aus der Affäre zu ziehen: servir et disparaître. Opfer und Verzicht, fürwahr ein edles Paar. Nur Neider würden dagegenhalten, dass disparaître auch heissen könnte «sich auf seine Pfründe zurückziehen». Nun ja, unser Grossmogul wird auch weiterhin Hof halten und seine gnädige Hand zum Gruss erheben. mengebiete. Im ersten Teil wird die aktuelle Situation der Hausärzte gegenüber dem Problem der geriatrischen Versorgung in den drei deutschsprachigen Ländern dargestellt. Der zweite Abschnitt widmet sich den spezifischen medizinischen Problemen, die bei älteren Patienten bedeutend sind. Im dritten Teil werden therapeutische Besonderheiten der geriatrischen Patienten betrachtet. Das vierte Kapitel gibt Hinweise zum Vorgehen bei Versorgungsproblemen.

Die Autoren gehen auf palliative Geriatrie und ethische Fragen ein. Der Patient und seine Angehörigen stehen dabei stets im Mittelpunkt. Ihre Interessen sollen verwirklicht werden. Im Anhang finden sich viele nützliche Assessment- und Fragebögen sowie Protokolle zur praktischen Anwendung. Anhand von Fallbeispielen werden konkrete Situationen, die immer wieder so oder ähnlich auftauchen, vorgestellt und praxisnah erläutert. Tabellen, Schemata und Skalen unterstützen das Verständnis, des in gut überschaubare Abschnitte gegliederten Textes. Das Buch vermittelt zwischen Hausärzten und Fachärzten anderer Spezialisierungen. Es zeigt deutlich, welchen Nutzen eine gute Zusammenarbeit für beide Seiten hat und insbesondere natürlich für den Patienten.

Sandra Krüger, Berlin 